\title{
Preoperative Visualization of the Artery of Adamkiewicz by Dual-Phase CT Angiography in Patients with Aortic Aneurysm
}

\author{
MAU AMAKO, YOSHIAKI YAMAMOTO*, KATSUMI NAKAMURA*, SATORU TOBINAGA, \\ EIJI NAKAMURA, YUKIO HOSOKAWA, TOMOKAZU OHNO, HIDETOSHI AKASHI, \\ SHIGEAKI AOYAGI** AND HIROYUKI TANAKA
}

\author{
Department of Surgery, Kurume University School of Medicine, Kurume 830-0011, \\ *Department of Radiology, Kyoaikai Tobata Kyoritsu Hospital, Kitakyusyu 804-0093, \\ and **Department of Cardiovascular Surgery, Munakata Suikoukai General Hospital, \\ Fukutsu 811-3298, Japan
}

Received 9 November 2011, accepted 16 April 2012

\begin{abstract}
Summary: To improve our ability to visualize the Adamkiewicz artery (AKA), we developed a modified intravenous CT angiography technique, which we refer to as right atrial CT (RA-CT) angiography. In this study, AKA detection rate and visualization of the arterial continuity from the aorta to the anterior spinal cord artery (ASA) was evaluated using RA-CT angiography.

We performed RA-CT angiography in 110 patients with abdominal, thoracic descending, or thoracoabdominal aortic aneurysms. In RA-CT angiography, contrast medium with a high iodine concentration $(370 \mathrm{mg} / \mathrm{dl})$ was injected twice into the right atrium at a high injection rate $(8.0 \mathrm{ml} / \mathrm{sec})$, and two CT scans, starting at $20 \mathrm{sec}$ after the first injection and at $35 \mathrm{sec}$ after the second injection, respectively, were performed. All CT images were obtained using an 8- or 16-detector CT scanner at a slice thickness of $0.625 \mathrm{~mm}$. The AKA was defined as the largest radiculomedullary artery with a characteristic hairpin turn, and with continuity from the aorta to the ASA.

The AKA with hairpin turn was detected in all patients (100\%), and continuity from the aorta to the ASA was confirmed in 99 of the 110 patients $(90.0 \%)$. The AKA arose between Th8 and L1 in 86 of these patients $(86.8 \%)$, and originated from the left side in 71 patients $(71.7 \%)$.

RA-CT angiography may be useful for visualizing the AKA and the arterial continuity from the aorta to the ASA in patients with aortic aneurysm, although the use of more advanced CT machines will provide safe and easy identification of the AKA and arterial continuity with a small amount of contrast medium and a single scan.
\end{abstract}

Key words spinal cord ischemia, paraplegia, CT angiography, Adamkiewicz artery, anterior spinal artery.

\section{INTRODUCTION}

Paraplegia and paraparesis due to spinal cord ischemia are the most serious complications after repair of thoracic descending and thoracoabdominal aortic aneurysms. Spinal cord ischemia has been reported to occur in $5-10 \%$ of patients who undergo operative repair of these aneurysms [1-4].

Blood supply to the spinal cord is primarily main- tained through the anterior spinal artery (ASA) in which blood flow is supplied via the radiculomedullary arteries arising from the segmental arteries such as the intercostal, the lumbar, or the subclavian artery. Among the radiculomedullary arteries, the most dominant artery in the thoracoabdominal region is called the Adamkiewicz artery (AKA). Accordingly, preoperative identification of the AKA, together with intraoperative reconstruction of the segmental artery from

Correspondence: Dr. Amako M, Department of Surgery, Kurume University School of Medicine, 67 Asahi-machi, Kurume 830-0011, Japan. Tel:+81 942 35 3311 Fax: +81 942358967 E-mail: amakomau@yahoo.co.jp

Abbreviations: AAA, abdominal aortic aneurysm; AKA, Adamkiewicz artery; ASA, anterior spinal artery; FOV, field of view; IA-CT, intra-arterial CT; IVCT, Intravenous CT; MR, magnetic resonance; RA-CT, right atrial CT; TAA, thoracic descending aortic aneurysm; TAAA, thoracoabdominal aortic aneurysm. 
which the AKA originates, is very important to minimize the risk of postoperative spinal cord ischemia.

Previous studies have shown that selective spinal cord angiography $[5,6]$, multi-detector row computed tomography (CT) angiography [7-11], and magnetic resonance (MR) angiography [12,13] are useful for identifying the AKA and the ASA preoperatively. However, the visualization of the AKA and of the continuity between the aorta and the ASA with these techniques may not be adequate to prevent spinal cord ischemia after surgery. To improve visualization of the AKA using CT angiography, we developed a modified intravenous CT angiography technique, which we refer to as right atrial CT (RA-CT) angiography. So far as we are aware, this is the first report of this technique.

In the present study, visualization of the AKA, and of the continuity between the aorta and the ASA using RA-CT angiography were assessed, and the branching level and laterality of the origin of the AKA were determined.

\section{MATERIALS AND METHODS}

Between January 2004 and June 2010, 110 patients with abdominal aortic aneurysm (AAA), thoracic descending aortic aneurysm (TAA) or thoracoabdominal aortic aneurysm (TAAA) underwent RA-CT angiography to identify the AKA and the ASA, as a preoperative procedure. The 110 patients included 87 male and 23 female patients who ranged in age from 23 to 85 years, with a mean age of $68.4 \pm 10.6$ years. The aneurysm was AAA in 8 of the 110 patients, TAA in 67 , and TAAA in 35 . As to etiology, the aneurysm was atherosclerotic in 61 patients and dissecting in 49. Clinical characteristics of the 110 patients were summarized in Table 1. The exclusion criteria for RA-CT angiography were a previous allergic reaction to contrast medium and renal insufficiency (serum creatinine $>2.0 \mathrm{mg} / \mathrm{dl}$ ). Informed consent was obtained from all patients after explaining the possible complications of $\mathrm{CT}$ angiography and the side effects of large doses of contrast medium.

For RA-CT angiography, a $5 \mathrm{~F}$ straight catheter (CATHEX, Tokyo, Japan) was inserted into the RA via the femoral vein under fluoroscopic guidance, after which the patient was transferred to a CT room. Contrast medium $(100 \mathrm{ml})$ containing $370 \mathrm{mg} / \mathrm{ml}$ iopamidol (Shering, Berlin, Germany) was injected into the $\mathrm{RA}$ at a rate of $8 \mathrm{ml} / \mathrm{sec}$ with a power injector (NemotoKyourindo, Tokyo, Japan), followed by a $20 \mathrm{ml}$ physiological saline solution flush at the same rate, and then a CT scan was performed in each patient. In our RA-CT
TABLE 1.

Patient Characteristics

\begin{tabular}{|c|c|c|}
\hline Variable & Years $($ Mean $\pm \mathrm{SD})$ & $\mathrm{N}(\%)$ \\
\hline Age (years) & $36-85(68.4 \pm 10.6)$ & \\
\hline Gender (male) & & 87 (79) \\
\hline Diabetes mellitus & & $18(16)$ \\
\hline Hypertension & & $96(87)$ \\
\hline Hyperlipidemia & & $23(21)$ \\
\hline CAD (by history or ECG) & & $14(13)$ \\
\hline Cerebrovascular disease & & $16(14)$ \\
\hline COPD & & $41(38)$ \\
\hline \multicolumn{3}{|l|}{ Aneurysm pathology } \\
\hline atherosclerotic & & $61(55)$ \\
\hline dissection & & $49(45)$ \\
\hline AAA & & $8(7)$ \\
\hline TAA & & $67(61)$ \\
\hline TAAA & & $35(32)$ \\
\hline \multicolumn{3}{|c|}{$\begin{array}{l}\mathrm{CAD}=\text { coronary artery disease }, \mathrm{COPD}=\text { chronic obstruc- } \\
\text { tivepulmonary disease, } \mathrm{AAA}=\text { abdominal aorthic aneurysm } \\
\text { TAA = thoracic aortic aneurysm, TAAA = tharaabdominal } \\
\text { aortic aneurysm }\end{array}$} \\
\hline
\end{tabular}

angiography protocol, injection of contrast medium and CT scan were performed twice to improve visualization of the AKA and of the arterial continuity, particularly in patients with hemodynamic disorders such as poor cardiac function, arrhythmias, bradycardia, or valve disease, and to obtain denser enhancement of the AKA in patients with a large aortic aneurysm or collateral arteries connecting with the ASA. The first CT scan was started $20 \mathrm{sec}$ after the commencement of the first injection of contrast. The second CT scan was begun $35 \mathrm{sec}$ after initiation of the second injection of contrast medium with the same injection protocol. Consequently, the scanning period for the opacified aorta and arteries feeding the spinal cord was expected to total $30 \mathrm{sec}$. The detailed RA-CT angiography procedures were schematically shown in Fig. 1.

For this study, an 8- or 16-row MDCT scanner (Lightspeed; GE Medical Systems, Milwaukee, USA) was used, and CT scans were obtained from the level of the first cervical vertebra to the inferior margin of the symphysis pubis. Shallow breathing was permitted for all patients throughout the scanning procedure. Each CT scan was performed using the following parameters: helical pitch, $1.375 ; 0.8 \mathrm{sec}$ per rotation; 120 $\mathrm{kV}$; 300-400 mA; calibration field of view (FOV), 500 $\mathrm{mm}$; display FOV, 160-210 mm; and image slice thick- 


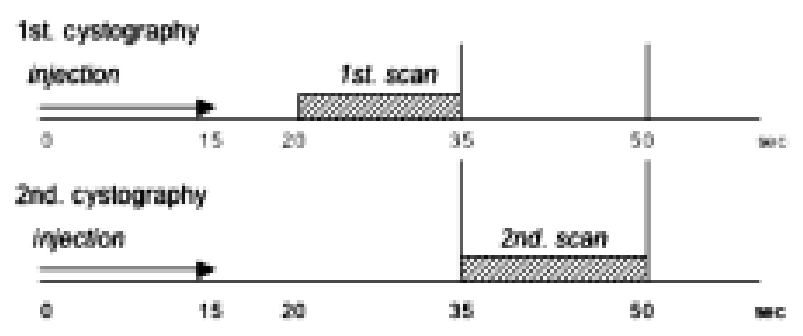

Fig. 1. Before each scan was started, $120 \mathrm{ml}$ of a contrast material containing saline was injected at a rate of $8.0 \mathrm{~mL} / \mathrm{sec}$. The scan delay after the start of the first injection was 20 seconds and after the second injection was 35 seconds.

ness, $0.625 \mathrm{~mm}$. Images were processed on a 3D workstation (Virtual place; AZE, Tokyo, Japan). Visualization of the hairpin turn and of the continuity between the aorta and the ASA were obtained by curved- and multiplanar reformations. All CT images were evaluated by one cardiovascular surgeon (S.T.) and 2 experienced radiologists (Y.Y, K.N) who were blinded to this study. In case of disagreement about the evaluation of an image, final consensus was reached through interobserver discussion. In this study, the diagnostic criteria for the AKA were both the presence of a so-called hairpin turn connecting to the ASA and the confirmation of the continuity of the aorta, the segmental artery such as the intercostal or lumbar artery, the AKA, and the ASA. Differentiation of the AKA from the anterior radiculomedullary vein is very difficult on the basis of their characteristic shapes; thus, the AKA was confirmed by visualizing the arterial continuity between the aorta and the ASA. The branching level of the AKA was determined on the basis of the anatomic level of the segmental artery from which the AKA originated, and the anatomic level of the segmental artery was defined as the level of the rib below which the segmental artery ran.

\section{STATISTICAL ANALYSIS}

Continuous data presented as mean \pm standard deviation. They were analyzed using a 2 -sided paired $t$ test. Data analysis was performed on JMP version 5.1 (SAS Institute, Inc, Cary, NC)

\section{RESULTS}

No complications related to the RA-CT angiography procedure such as paraplegia, bleeding, or aneurysm rupture were experienced, however, renal impairment caused by contrast medium (elevation of serum creatinine $\geq 0.5 \mathrm{mg} / \mathrm{dl}$ from the baseline creatinine value before the procedure) was observed in one patient, who was successfully managed with conservative treatment. The obtained data could be analyzed in all cases.

The AKA with hairpin turn was detected in all 110 patients $(100 \%)$, and visualization of the continuity between the aorta and the ASA was confirmed in 99 patients (90.0\%), as shown in Table 2 and Fig. 2. The arterial continuity was not traceable in the remaining 11 patients $(10.0 \%)$. Of these 11 patients, $5(45.5 \%)$ had a dissecting aortic aneurysm with very slow blood flow in the false lumen, and 7 (63.7\%) had a large aneurysm with massive mural thrombi leading to occlusion of an ostium of the intercostal or lumbar artery. On scan timing, the AKA and the arterial continuity were visualized in the first scan (20 to $35 \mathrm{sec}$ after beginning the injection of contrast medium) in $81(81.8 \%)$ of the 99 patients and were observed in the second scan (35 to $50 \mathrm{sec}$ after beginning the injection) in 18 $(18.2 \%)$, as shown in Table 2. Among these 18 patients, $13(72.2 \%)$ had a dissecting aneurysm. With regard to aneurysm etiology, the AKA and arterial continuity were observed in $61(100 \%)$ and $55(90.2 \%)$ of the 61 patients with a true aneurysm, respectively, and in 49 $(100 \%)$ and $44(89.8 \%)$ of the 49 patients with a dissecting aortic aneurysm, respectively. The difference in the detection rate of the AKA or visualization of the arterial continuity between these two patient-groups

TABLE 2

Detection rate on etiology

\begin{tabular}{lcc}
\hline & Detection rate of the AKA & Detection rate of continuity \\
\hline All patients & $110(100 \%)$ & $99(90 \%)$ \\
Aortic etlology & & \\
True aneurysm & $61(100 \%)$ & $55(90.2 \%)$ \\
Dissection & $49(100 \%)$ & $44(89.8 \%)$ \\
\hline
\end{tabular}


A

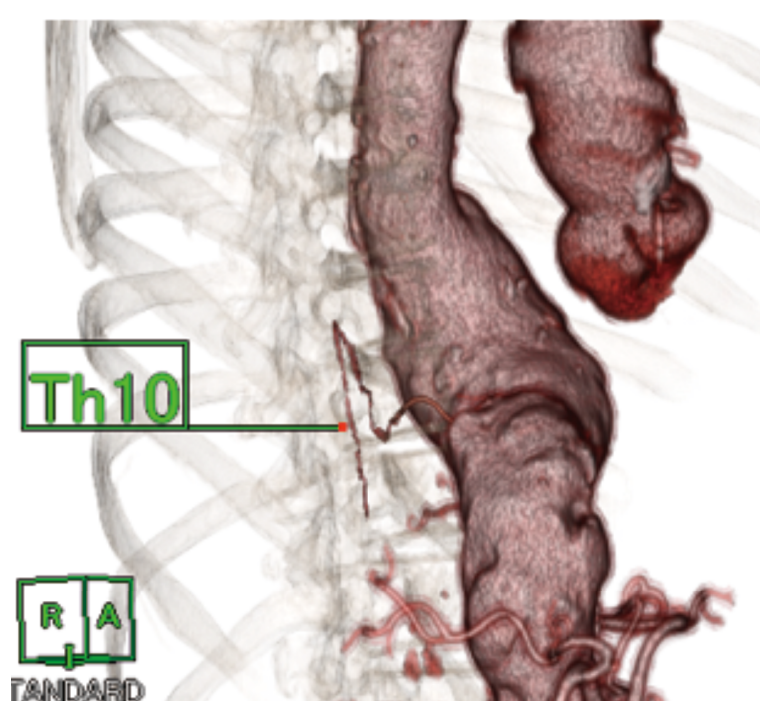

B

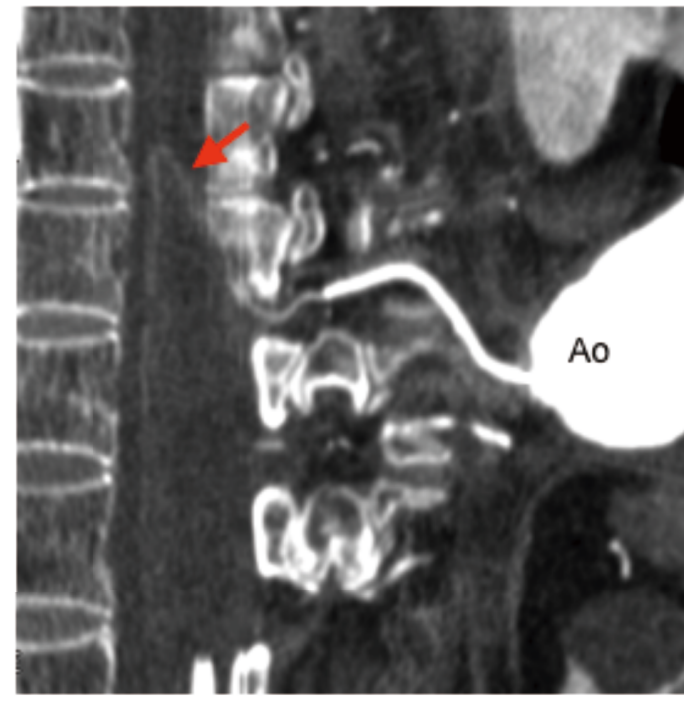

Fig. 2. One radicular artery. A) The right anterior oblique three-dimensional VR image. B) A 2D curved-MPR demonstrates connection from aorta to anterior spinal artery by the AKA, and its continuity. Red arrow points to AKA. Th10=10th thoracic vertebra, Ao=aorta, VR: volume rendering, MPR; multi planar reconstruction

TABLE 3.

Origins of AKA in 99 patients

\begin{tabular}{|c|c|c|c|c|c|c|}
\hline Level of AKA origin & Left & Right & No. & $\%$ & & \\
\hline TH6 & 1 & 0 & 1 & 1.0 & \multirow{11}{*}{$86.8 \%$} & \multirow{11}{*}{$95.0 \%$} \\
\hline Th7 & 1 & 0 & 1 & 1.0 & & \\
\hline Th8 & 8 & 2 & 10 & 10.1 & & \\
\hline Th9 & 14 & 8 & 22 & 22.2 & & \\
\hline Th10 & 18 & 1 & 19 & 19.2 & & \\
\hline Th11 & 6 & 5 & 11 & 11.1 & & \\
\hline Th12 & 8 & 3 & 11 & 11.1 & & \\
\hline L1 & 7 & 6 & 13 & 13.1 & & \\
\hline $\mathrm{L} 2$ & 7 & 1 & 8 & 8.0 & & \\
\hline L3 & 1 & 1 & 2 & 2.0 & & \\
\hline \multirow[t]{2}{*}{ L4 } & 0 & 1 & 1 & 1.0 & & \\
\hline & $71(72 \%)$ & $28(28 \%)$ & $99 / 99$ & & & \\
\hline
\end{tabular}

was not significant $(\mathrm{p}=0.9719)$.

Among the 99 patients in whom the AKA and the arterial continuity were confirmed by RA-CT angiography, the segmental artery from which the AKA arose originated from the Th8 to L2 level in the aorta in 94 patients $(95.0 \%)$, and particularly between Th8 and L1 in 86 patients $(86.8 \%)$, as shown in Table 3 . The segmental artery connecting with the AKA originated on the left side in 71 patients $(71.7 \%)$ and on the right side in 28 patients $(28.3 \%)$. Of the 99 patients, $79(79.8 \%)$ had a single AKA, however, multiple radiculomedullary arteries supplying the AKA were found in 20 patients $(20.2 \%)$. In 19 of these 20 patients, 2 radiculomedullary arteries were recognized (Fig. 3-A) and 4 radiculomedullary arteries were observed in 1 patient (Fig. 3-B).

The presence of collateral circulation resulting from occlusion of the intercostal or lumbar arteries was depicted in $3(2.7 \%)$ of the 110 patients. The intercostal artery was the source of collateral vessels to the AKA 
A

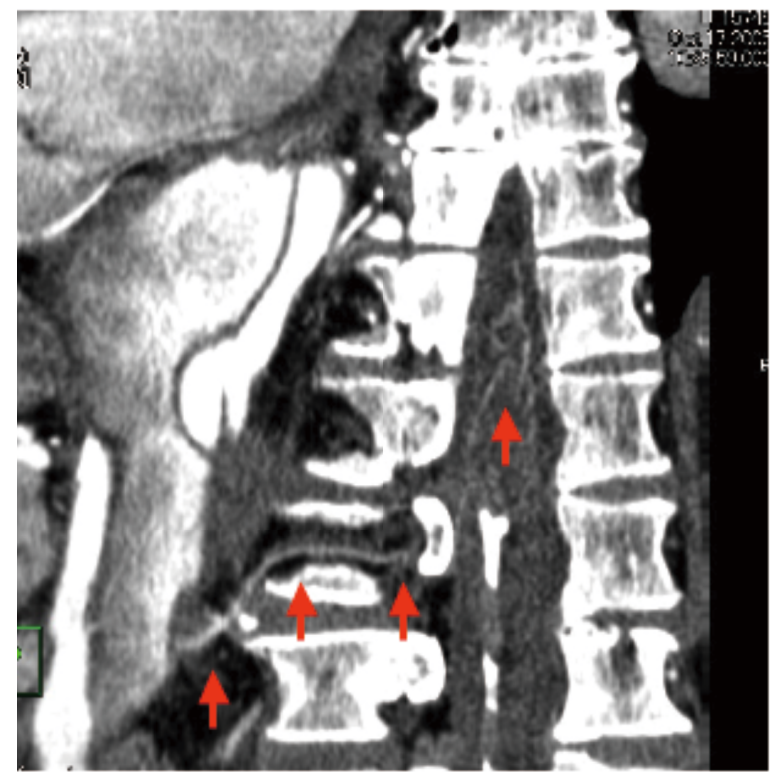

B

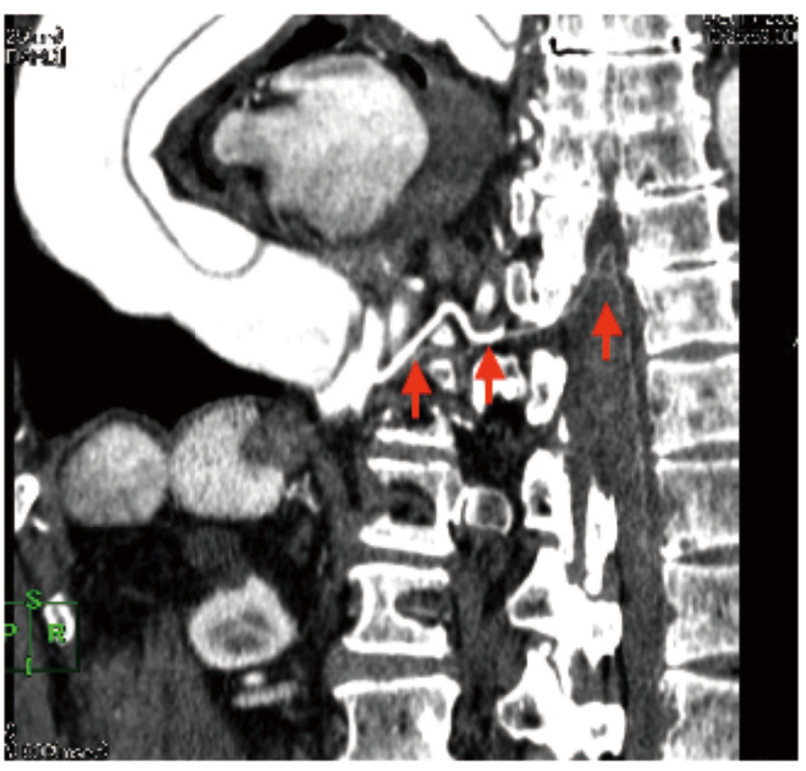

Fig. 3. 2D curved MPR images obtained at the 1st scan (A), and at the 2nd scan (B) in the same patient. Red arrow indicates the artery connecting the aorta to the anterior spinal artery. The 2nd scan image (B) showed higher enhancement of the AKA than the 1st scan image (A). MPR; multi planar reconstruction

A

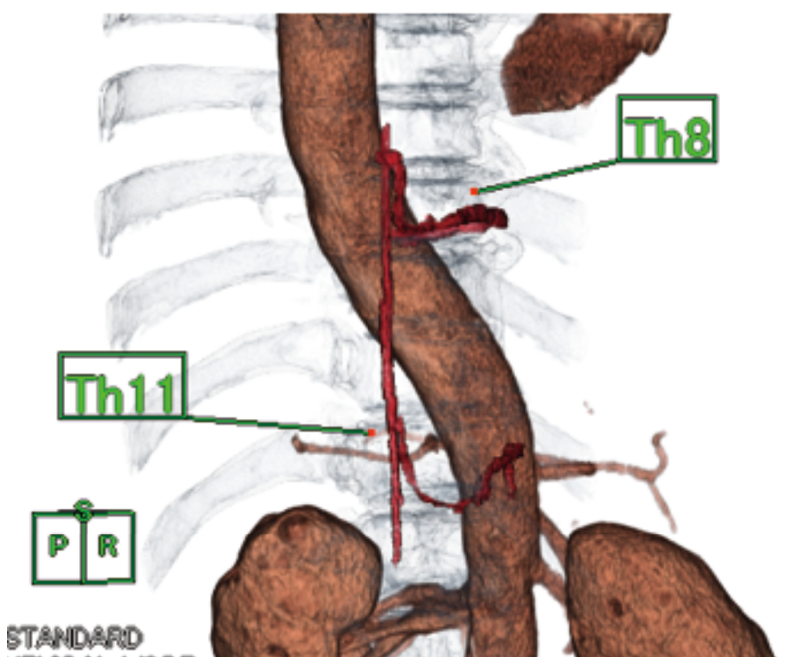

B

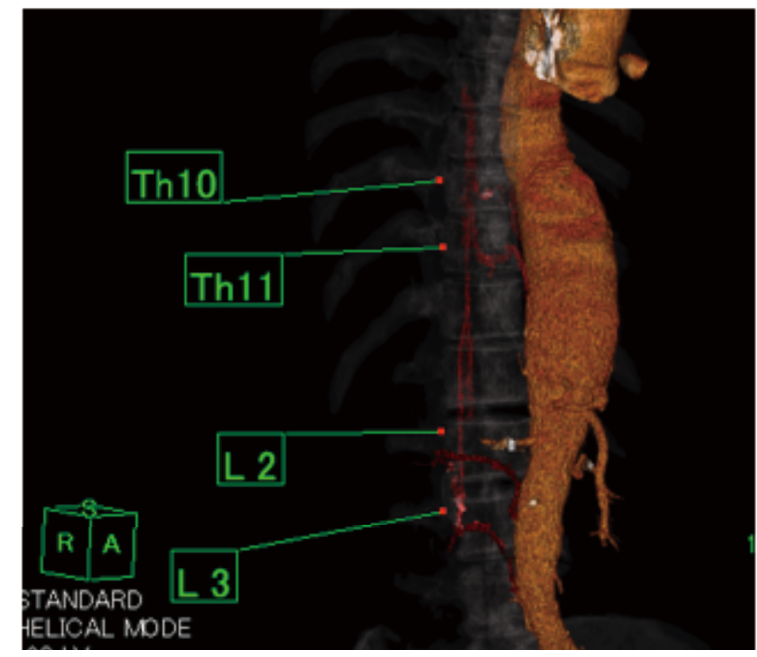

Fig. 4. A) Two radicular arteries. The right posterior oblique three-dimensional VR image. Two radicular arteries join the anterior spinal artery (Th8, Th11)

ICA=intercostals artery, Th8=8th thoracic vertebra, Th1111th thoracic vertebra, VR: volume rendering

B) Four radicular arteries. The right anterior oblique three-dimensional VR image. Th10=10th thoracic vertebra, Th11=11th thoracic vertebra, L2=2nd lumbar vertebra, L3=3rd lumbar vertebra, VR: volume rendering.

in 2 of the 3 patients. Collateral circulation was shown from the 11th to 9th intercostal artery (Fig. 4-A), from the 1st lumbar artery to 12th intercostal artery, and from the posterior spinal artery to the anterior spinal artery in one patient each. 


\section{DISCUSSION}

Spinal cord ischemia resulting in paraplegia and paraparesis is the most serious complication that can occur after thoracic descending and thoracoabdominal aortic aneurysm repair, and is mainly caused by interruption of blood supply to the spinal cord during the aortic operation. The ASA, which feeds the anterior two thirds of the spinal cord, is supplied from the AKA and is the major supplier of blood to the spinal cord in the thoracolumbar region. Therefore, preoperative visualization of the AKA and the ASA, and intra-operative reconstruction of the segmental artery from which the AKA arises, may help reduce the risk of postoperative spinal cord ischemia.

For preoperative identification of the AKA and the ASA, several angiographic techniques including selective spinal cord angiography [5,6], MR angiography $[12,13]$, and CT angiography [7-11] have been used. Conventional selective spinal cord angiography is an
A

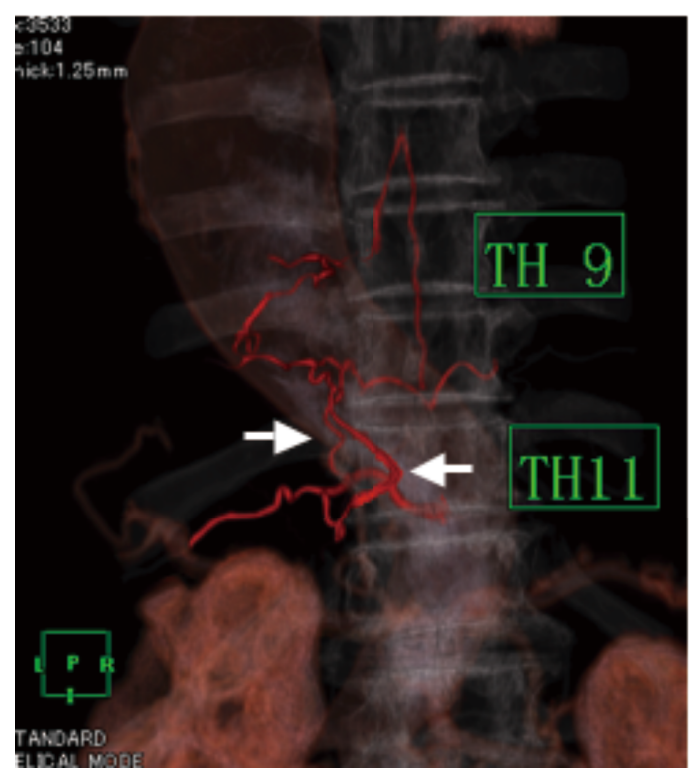

B

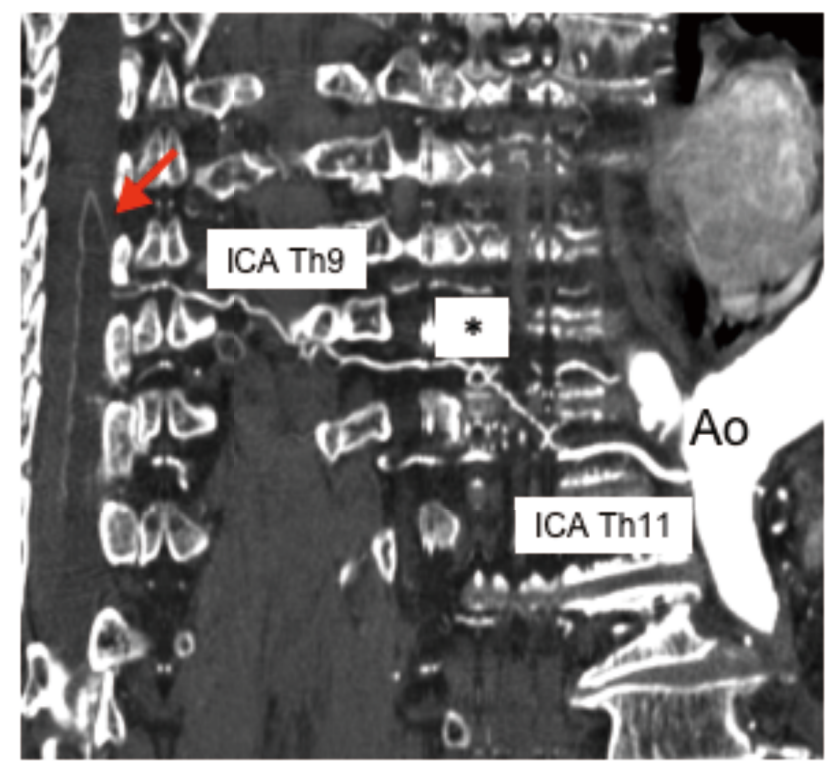

Fig. 5. Demonstration of collateral circulation to the AKA. A) The posterior inferior oblique threedemensional dimensional VR image. ICA9 is occluded proximally. It shows collateral circulation (white arrow) from the ICA11 to the ICA 9.

B) A 2D curved-MPR demonstrates connection from aorta to anterior spinal artery by the AKA, and its continuity through the collateral circulation $(*)$. Red arrow points to AKA. ICA=intercostals artery, Th9=9th thoracic vertebra, Th1 $1=$ th1 1 thoracic vertebra, Ao=aorta, VR; VR: volume rendering, MPR; multi planar reconstruction

TABLE 4.

Detection rates of the AKA by IV-CTA, SCA, IA-CTA and RA-CTA

\begin{tabular}{|c|c|c|c|c|c|c|c|}
\hline & Method & No. patients & $\begin{array}{c}\text { Deteclor } \\
\text { rows }\end{array}$ & $\begin{array}{l}\text { Slice } \\
\text { thickness } \\
(\mathrm{mm})\end{array}$ & $\begin{array}{c}\text { Detection } \\
\text { rate }(\%) \\
\text { hairpin turn }\end{array}$ & $\begin{array}{l}\text { Detection } \\
\text { rate }(\%) \\
\text { confinity }\end{array}$ & $\begin{array}{l}\text { injection } \\
\text { rate } \\
(\mathrm{ml} / \mathrm{sec})\end{array}$ \\
\hline Takase K et al, 2002 ${ }^{9)}$ & IV-CTA & 70 & 4 & 2 & 90 & 29 & 3.5 \\
\hline Yoshioka K et al, 2006 & IV-CTA & 30 & 16 & 0.5 & 83 & 60 & 3.5 \\
\hline Utsunomiya D et al, 2008 ${ }^{11)}$ & IV-CTA & 20 & 64 & 0.5 & 80 & 50 & 5.0 \\
\hline Kieffer E et al, 20025) & SCA & 480 & - & - & 91 & 83 & - \\
\hline Uotani $\mathrm{K}$ et al, 2009 & IA-CTA & 23 & 16 & 0.75 & 91 & 83 & - \\
\hline Present study & RA-CTA & 110 & 8 or 16 & 0.625 & 100 & 90 & 8.0 \\
\hline
\end{tabular}

SCA; spinal cord angiography, RA-CTA; night arid CT angiography 
effective and established technique, and the detectability of the AKA has been reported to be $43 \%$ to $86 \%$ $[5,6]$. This technique, however, is invasive, time-consuming, technically difficult to perform, and may be hazardous. Kieffer et al. [5] reported that major complications developed in $6(1.2 \%)$ of 480 patients, including paraplegia in 2 patients. With recent advances in MR angiography and CT angiography technologies, $\mathrm{CT}$ angiography and MR angiography have gained acceptance as effective and noninvasive alternative methods that allow visualization of the AKA and the ASA. According to several previous reports [12,13], the AKA was detected in $69 \%$ to $84 \%$ of patients by MR angiography without any complications. Furthermore, the use of both MR angiography and CT angiography can provide a very high detection rate of $90 \%$ for the AKA, which is superior to that of conventional selective spinal cord angiography [8]. However, compared with $\mathrm{CT}$ angiography, a more limited field of view is a major disadvantage in MR angiography [8]. Therefore, MR angiography may fail to depict the clinically important collateral vessels to the AKA in some patients, when a collateral source is the internal thoracic artery or the thoracodorsal artery $[8,15,16]$.

In CT angiography, contrast medium was principally administered intravenously or intra-arterially to opacify the AKA and the ASA. Intravenous CT (IV-CT) angiography, in which contrast medium is injected into a peripheral vein such as the antecubital vein, is noninvasive and easy to perform. Takase et al. [9] examined 70 patients with suspected thoracoabdominal vascular disease using $100 \mathrm{ml}$ contrast medium $(300 \mathrm{mg} /$ $\mathrm{ml}$ ) injected at $3.5 \mathrm{ml} / \mathrm{sec}$ and $2 \mathrm{~mm}$ thick CT sections, and identified the AKA in $63(90 \%)$ of patients. The AKA was successfully visualized in $25(83 \%)$ of 30 patients with 1-mm thick, 4-row CT angiography in a study by Yoshioka et al. [8]. For these CT scans, a total of $2.5 \mathrm{ml} / \mathrm{kg}$ of contrast medium $(370 \mathrm{mg} / \mathrm{ml})$ was injected at $3 \mathrm{ml} / \mathrm{sec}$. After injecting $100 \mathrm{ml}$ of contrast medium $(350 \mathrm{mg} / \mathrm{ml})$ at $5 \mathrm{ml} / \mathrm{sec}$, Kudo et al. [10] reported $68 \%$ AKA detection on arterial phase abdominal CT scans ( $2 \mathrm{~mm}$ section thickness) in 19 patients with liver disease but without known thoracic aortic disease. In these studies, the reported detection rate of the AKA has been relatively high, however, visualization of the arterial continuity from the aorta to the ASA was achieved in only $32 \%$ to $60 \%$ of patients [8-10]. Considering that confirmation of the arterial continuity is surgically more important than identification of the AKA and the ASA for minimizing postoperative spinal cord ischemia, visualization of the arterial continuity using IV-CT angiography may not be satisfac- tory in thoracic descending and thoracoabdominal aortic aneurysm repair. Such low visibility of the arterial continuity is generally a major disadvantage in IV-CT angiography. On the other hand, intra-arterial CT (IA-CT) angiography, where contrast medium is injected into the descending thoracic aorta, has an advantage because of higher arterial enhancement compared with IV-CT angiography. Uotani et al. [14] reported visualization of the AKA in $21(91.3 \%)$ of 23 patients and confirmation of the arterial continuity in $19(82.6 \%)$ of the 23 patients. IA-CT angiography, however, is invasive. Furthermore, difficulty in determining the proper scan timing is a major disadvantage in IA-CT angiography because of the short duration of arterial enhancement.

The quality of CT angiographic images is influenced by many factors, including the imaging parameters of the CT machine, the method of injecting contrast medium, and the imaging delay. Utsunomiya et al. [11] applied 64-row MDCT for identifying the AKA. In their study, no improvement in the detection rate of the AKA was observed despite an increase in the number of detector rows, however, the traceability of the AKA was superior at a $0.5-\mathrm{mm}$ slice thickness compared with a 2-mm slice thickness [11]. Since the AKA is approximately $1 \mathrm{~mm}$ in diameter, a slice thickness of 0.5 to 1 $\mathrm{mm}$ may be adequate for detection of the AKA.

It is well known that osseous structures sometimes interfere with visualization of arteries. Even when the osseous structures are normal, it may be difficult to visualize the artery that runs into an intervertebral foramen because not only is the artery very thin, it also runs close to the osseous structures. To improve the visualization of the AKA and the arterial continuity, it is essential to increase the contrast-to-noise ratio of a CT scan by both increasing arterial enhancement and decreasing the noise by anatomical structure [7]. Utsunomiya et al. [11] investigated a protocol for injecting contrast medium to obtain higher quality CT angiographic images, and have demonstrated that the injection protocol with a higher iodine concentration $(350 \mathrm{mg} / \mathrm{ml})$ and a faster injection rate $(5 \mathrm{ml} / \mathrm{sec})$ is significantly superior to that with a lower iodine concentration $(300 \mathrm{mg} / \mathrm{ml})$ and a slower injection rate $(3.5$ $\mathrm{ml} / \mathrm{s}$ ) for obtaining sufficient vascular enhancement and visualization of the AKA. Their protocol improved the detection rate of the AKA from 50\% to $80 \%$.

Both detection rate of the AKA and visualization of the arterial continuity in our RA-CT angiography were higher that those in conventional IV-CT angiography. In our method, contrast medium containing a high iodine concentration $(370 \mathrm{mg} / \mathrm{dl})$ was injected at 
a high injection rate $(8 \mathrm{ml} / \mathrm{sec})$, and moreover, it was directly administered into the RA. This injection protocol is expected to lead to higher arterial enhancement than the conventional injection protocol (3.5-5.0 $\mathrm{ml} / \mathrm{sec}$ ), in which contrast medium was injected into a peripheral vein. Our injection protocol may reduce dilution of contrast medium in the right heart and the lung, and may also work as a bolus injection of contrast medium. This modification of the contrast medium injection protocol may have been a major factor in enabling us to achieve not only very high detection rates of the AKA and the ASA, but also visualization of the arterial continuity in $90.0 \%$ of the patients, in our CT angiography.

On the other hand, the quality of CT angiographic images is also influenced by many hemodynamic factors, including cardiac function, heart rate, aneurysm size, and the presence of arrhythmia or valve disease in patients. In addition, the AKA and the ASA were enhanced through collateral vessels in some patients $[15,16]$. In these patients, visualization of the AKA and the ASA may be difficult when an automatic triggering system for the scan delay is employed because contrast enhancement of the AKA and the ASA may be slow and weak. Yoshioka et al. [8] have indicated that depiction of the AKA and the start of scanning at the optimal timing to obtain a good image in CT angiography are very difficult in patients with a dissecting aneurysm because blood flow in the false lumen is generally very slow. In our RA-CT angiography, two CT scans starting at $20 \mathrm{sec}$ after commencement of the first injection and at $35 \mathrm{sec}$ after initiation of the second injection, respectively, were performed for $15 \mathrm{sec}$ each. Consequently, the contrast enhanced arterial continuity from the aorta to the AKA, and the ASA was scanned for a total of $30 \mathrm{sec}$. This long scan duration may provide better visibility of the arterial continuity in some patients with slow blood flow, particularly patients with collateral circulation to the AKA such as patients with a dissecting aneurysm. Although no statistically significant difference was found in the detectability of the AKA and the arterial continuity between patients with a true aneurysm and those with a dissecting aneurysm in our study, the AKA and the arterial continuity were depicted plainly in the second scan in $18(18.2 \%)$ of the 99 patients, as compared with $81(81.8 \%)$ in the first scan. This long scan duration may also be one of factors responsible for the high detection rate of the AKA and the arterial continuity in our RA-CT angiography.

In our experience, visualization of arterial continuity was most difficult in patients with a dissecting aneurysm ( 5 cases) or patients with a large aneurysm containing massive mural thrombi (7 cases). However, no statistically significant differences were found in the mean maximal diameter of the aneurysm $(\mathrm{p}=0.834)$ and aortic pathology (true aneurysm or dissecting aneurysm) regardless of whether the arterial continuity was visualized or not.

In this study, the level of origin of the AKA ranged from Th8 to L2 in $95.0 \%$ and the left side origin was $71.7 \%$. These results were in agreement with those of previous reports including anatomic studies, MR angiography, and CT angiography [7-12].

Although RA-CT angiography achieved a high visualization rate of the AKA and of the arterial continuity from the aorta to the ASA, there are some drawbacks. Dual-phase injection and scan procedures in this technique increase the patient's exposure to radiation and the dosage of contrast medium. The use of a total of $200 \mathrm{ml}$ of the contrast medium may be clinically problematic for patients with inherent atherosclerotic disease because they sometimes have marginal renal functions. For such patients, a single biphasic injection of contrast medium at a rapid injection rate might be helpful for depiction of the AKA and preservation of renal function [17]. Furthermore, RA-CT angiography is time-consuming and troublesome because of the need to transfer patients to a CT room after catheter insertion in a fluoroscopy room. The use of a flowdirected catheter might simplify RA-CT angiography procedures without requiring patient transfer or use of a fluoroscope. Finally, an early model CT scanner was used in this study, however, the use of more advanced CT machines will provide safe and easy identification of the AKA and arterial continuity with a small amount of contrast medium and a single scan.

Our study has several limitations. First, it is inherently limited by its retrospective nature. Second, the lack of a reference standard, such as conventional IV-CT angiography and IA-CT angiography, to evaluate the usefulness of RA-CT angiography is another drawback. Third, although this study included a relatively large number of patients (over 100) with aortic aneurysm, even this number of patients may be not sufficient to draw a firm conclusion about the role of this RA-CT angiography. Fourth, we gave the patients double the standard radiation dose and volume of contrast medium, compared with the usual method. Further study is needed to clarify the usefulness of RA-CT angiography in visualizing the AKA in patients with aortic aneurysm.

In conclusion, this study demonstrated that RA-CT angiography could achieve a very high detection rate 
of the AKA and visualization of the arterial continuity without any complications in patients with aortic aneurysm. These results suggest that RA-CT angiography is a safe and effective technique for identifying the AKA and the ASA and for visualization of the arterial continuity between the aorta and the AKA.

\section{REFERENCES}

1. Cambria RP, Clouse WD, Davison JK, Dunn PF, Corey M et al. Thoracoabdominal aneurysm repair: results with 337 operations performed over a 15-year interval. Ann Surg 2002; 236:471-479.

2. Estrera AL, Miller CC 3rd, Huynh TT, Porat E, and Safi HJ. Neurologic outcome after thoracic and thoracoabdominal aortic aneurysm repair. Ann Thorac Surg 2001; 72:1225-1231.

3. Hollier LH, Money SR, Naslund TC, Proctor CD Sr, Buhrman WC et al. Risk of spinal cord dysfunction in patients undergoing thoracoabdominal aortic replacement. Am J Surg 1992; 164:210-213.

4. Coselli JS, LeMaire SA, Miller CC 3rd, Schmittling ZC, Köksoy $\mathrm{C}$ et al. Mortality and paraplegia after thoracoabdominal aortic aneurysm repair: a risk factor analysis. Ann Thorac Surg 2000; 69:409-414.

5. Kieffer E, Fukui S, Chiras J, Koskas F, Bahnini A et al. Spinal cord arteriography: a safe adjunct before descending thoracic or thoracoabdominal aortic aneurysmectomy. J Vasc Surg 2002; 35:262-268.

6. Sadaver SJ, Williams GM, Trerotola SO, Perler BA, Wang $\mathrm{MC}$ et al. Preoperative spinal cord artery localization and its relationship to postoperative neurologic complications. Radiology 1993; 189:165-171.

7. Zhao SH, Logan L, Schraedley P, and Rubin GD. Assessment of the anterior spinal artery and the artery of Adamkiewicz using multi-detector CT angiography. Chin Med J 2009; 122:145-149.

8. Yoshioka K, Niimuma H, Ehara S, Nakajima T, Nakamura $\mathrm{M}$ et al. MR angiography and CT angiography of the artery of Adamkiewicz: state of art. Radiographics 2006; 26:S63S73.

9. Takase K, Sawamura Y, Igarashi K, Chiba Y, Haga K et al. Demonstration of the artery of Adamkiewicz at multidetector row helical CT. Radiology 2002; 223:39-45.

10. Kudo K, Terae S, Asano T, Oka M, Kaneko K et al. Anterior spinal artery and artery of Adamkiewicz detected by using multi-detector row CT. Am J Neuroradiol 2003; 24:13-17.

11. Utsunomiya D, Yamashita Y, Okumura S, and Urata J. Demonstration of the Adamkiewicz artery in patients with descending or thoracoabdominal aortic aneurysm: optimization of contrast-medium application for 64-detector-row CT angiography. Eur Radiol 2008; 18:2684-2690.

12. Hyodoh H, Kawaharada N, Akiba H, Tamakawa M, Hyodoh $\mathrm{K}$ et al. Usefulness of preoperative detection of artery of Adamkiewicz with dynamic contrast-enhanced MR angiography. Radiology 2005; 236:1004-1009.

13. Yamada N, Takamiya M, Kuribayashi S, Okita Y, Minatoya $\mathrm{K}$ et al. MRA of the Adamkiewicz artery: a preoperative study for thoracic aortic aneurysm. J Comput Assist Tomogr 2000; 24:362-368

14. Uotani K, Yamada N, Kono A, Taniguchi T, Sugimoto K et al. Preoperative visualization of the artery of Adamkiewicz by intra-arterial CT angiography. J Jpn Coll Angiol 2007; 47:511-517.

15. Nakai M, Shimizu S, Ochi Y, Kato G, and Okada M. Thoracodorsal artery as a collateral source to the artery of Adamkiewicz after endovascular aneurysm repair for descending thoracic aortic aneurysm. Eur J Endocasc Surg 2009; 37:566-568.

16. Yoshioka K, Niinuma H, Kawazoe K, and Ehara S. Threedimensional demonstration of the collateral circulation to the artery of Adamkiewicz via internal thoracic artery with 16-row multi-slice CT. Eur J Cardio-thorac Surg 2005; 28:492.

17. Fleischmann D, Rubin GD, Bankier AA, and Hittmair K. Improved uniformity of aortic enhancement with customized contrast medium injection protocols at CT angiography. Radiology 2000; 214:363-371. 